\title{
Editorial
}

\section{How big a challenge to initiate three basic infection control practices in hospitals of Bangladesh?}

Basic infection control practices in a hospital consist of a few simple common sense based activities which include hand hygiene, safe waste disposal and isolation of patients infected by dangerous organisms. Other activities of infection control program include surveillance of infection, training of staff, preparation and use of antibiogram, quality assurance of central sterile supply department, management of accidental needle stick injury, vaccination of staff etc.

If we look at the history, the formal infection control program started in the latter half of last century when CDC (center for disease control) of United States came forward with a recommendation that each hospital will appoint an infection control nurse and an epidemiologist to prevent, control and estimate infections in the hospitals ${ }^{1}$. Soon (1976) this was followed by publication of standards set by JCAHO (Joint Commission on Accreditation of Health Care Organizations) as a condition for hospital accreditation ${ }^{2}$. Thereafter, for accreditation, hospitals adopted infection control program and continued to improve upon it. Presently this program became an integral part of a hospital management in developed countries. Unfortunately almost forty years have passed since the recommendations of CDC was published and we have not even initiated basic infection control practices leave alone the other activities mentioned above.

Let us discuss on these three basic practices which can easily be interwoven into our existing hospital management system.

Since healthcare associated infection (HAI) is transmitted via hands of healthcare workers, as experimented by Ignaz Semmelweis in 1846, hand washing remains the single most important means to prevent infection ${ }^{3,4}$. Alcohol based hand rub is a very effective and popular hand washing material now a days. However, soap should be used when hands are visibly dirty, contaminated by body fluid or handling patients with colitis caused by Clostrium difficille. It should not be too difficult for any hospital to provide $70 \%$ ethyl alcohol and soap to healthcare workers (HCW) for disinfecting their hands before and after touching patients. It only needs training and motivation. The benefit derived from hand hygiene protocol undoubtedly overweighs the cost of alcohol and soap.
Patients harboring dangerous organisms should be placed in separate rooms. This is essential to protect other patients and healthcare workers. Most of our hospitals do not follow isolation protocols because of lack of awareness as well as faults in their structural design. Hospitals should be built according to recommended design which includes provision for contact, droplet and airborne precautions and provision for positive pressure rooms for immunocompromised patients and negative pressure rooms for air borne precaution ${ }^{5,6}$. It is not too difficult to create isolation facilities in hospitals where such provisions are presently nonexistent. A corner room or two in each floor can be converted into isolation area with provisions for personal protective gears such as gown, gloves, mask, etc.

In Bangladesh management of hospital waste is another neglected area where there is gross violation of basic human rights ${ }^{7}$. In general, hospitals do not have a system for safe disposal of wastes. Recycling of discarded items from hospitals and clinics is common topic in the media coverage. Sorting the hospital wastes by categories of sharps, infected and non infected items and disposing them to recommended place through proper processing should be considered as one of the basic necessities of hospital management.

In Dhaka some of the privately run hospitals have initiated basic infection control measures but the overwhelming majority of both government and private hospitals are even not aware of existence of such measures. Microbiologists can play a key role here. They can contribute not only by providing culture and sensitivity reports but also by helping in conducting training program for healthcare workers, quality control of central sterile supply department (CSSD), and surveillance.

There are a few voluntary organizations who are working on infection control in Bangladesh. Infection Control and Prevention in Bangladesh (ICPPB) is one of them who has initiated conducting seminars and discussions at hospitals and health institutes of Dhaka city. It is encouraging to observe that such endeavors have brought some positive changes in private sectors. But the response from government is far from satisfactory. It is high time the government should come forward and implement the basic infection control practices in all hospitals; and the sooner the better. 
Prof. Zahidul Hasan

Consultant

Microbiology and Head, Infection control program, Square Hospitals Ltd 18/F West Panthapath, Dkaka, Bangladesh

Email: Zahidul@squarehospital.com

\section{References}

1. Garner JS, Bennett JV, Scheckler WE, Maki DG, Brachman PS. Surveillance of nosocomial infections. In: Centers for Disease Control. Proceedings of the International Conference on Nosocomial Infections. Atlanta (GA): Centers for Disease Control; 1970. p. 27781.

2. Joint Commission on Accreditation of Hospitals. Accreditation Manual for Hospitals. Chicago (IL): Joint Commission on Accreditation of Hospitals; 1976.

3. Bauer TM, Ofner E, Just HM, et al. An epidemiological study assessing the relative importance of air borne and direct contact transmission of microorganisms in a medical intensive care unit. J Hosp Infect 1990;15: 301309

4. Semmelweis I. Etiology, concept, and prophylaxis of childbed fever. Carter KC, ed. 1st ed. Madison, WI: The University of Wisconsin Press, 1983.

5. AIA 2001. Guidelines for Design and Construction of Hospital and Health Care Facilities:2001 Edition. New York, NY: American Institute of Architects/Facility Guidelines Institute.

6. Siegel JD, Rhinehart E, Jackson M, Chiarello L and Healthcare Infection Control Practices Advisory Committee, 2007. Guideline for Isolation Precautions: Preventing Transmission of Infectious Agents in Healthcare Settings, June 2007

7. Zerin SA, Ahmed MB. Hospital waste management in Dhaka: a threat. Bangladesh Res. Pub.J; 3(1): 796-811 